\title{
Criminologie
}

\section{Victimologie : tendances récentes}

\section{Ezzat Fattah}

Volume 13, numéro 1, 1980

Regards sur la victime

URI : https://id.erudit.org/iderudit/017114ar

DOI : https://doi.org/10.7202/017114ar

Aller au sommaire du numéro

Éditeur(s)

Les Presses de l'Université de Montréal

ISSN

0316-0041 (imprimé)

1492-1367 (numérique)

Découvrir la revue

Citer cet article

Fattah, E. (1980). Victimologie : tendances récentes. Criminologie, 13(1), 6-36. https://doi.org/10.7202/017114ar d'utilisation que vous pouvez consulter en ligne.

https://apropos.erudit.org/fr/usagers/politique-dutilisation/ 
Les dernières années ont témoigné des développements importants dans le domaine de la victimologie. L'étude des victimes du crime, qui durant les années cinquante et soixante était à l'état embryonnaire, a fait des grands pas en avant, et, bien qu'on puisse difficilement prétendre que la victimologie a atteint le statut d'une discipline autonome, elle a sans doute affirmé sa présence en tant que branche prometteuse de la criminologie. Présentement, la victimologie fait face à certaines attaques et critiques similaires à celles qu'on a adressées, il y a quelques décennies, à la criminologie, mais elle progresse lentement vers la maturité scientifique et acquiert de plus en plus la respectabilité académique. Dans le monde entier, elle est reconnue actuellement comme partie intégrante de la science criminologique. Plusieurs facteurs et événements en sont responsables : a) un corps de connaissance qui ne cesse de croître et une littérature grandissante sur les victimes du crime et sur la victimisation ; b) une série de symposia internationaux de victimologie commencée en 1973 ; c) un symposium international de victimologie tenu à Bellagio, en Italie, en 1975 ; d) une revue scientifique internationale consacrée uniquement à la victimologie et publiée à Washington, D.C., par Visage Press.

Même si la victimologie, en tant que discipline scientifique, est encore dans son enfance, il serait présomptueux de vouloir faire ici le bilan de ses progrès théoriques ou de tenter une revue exhaustive de ses réalisations pratiques. Le but de cet article est donc de discuter de l'état actuel de la victimologie, d'examiner et d'analyser quelques tendances récentes et quelques développements nouveaux autant sur le plan théorique qu'appliqué.

Selon nous, la victimologie a subi récemment une certaine métamorphose. Elle s'est transformée d'une *victimologie de l'acte » en une " victimologie d'action ». Initialement, la victimologie était essentiellement axée sur des délits spécifiques tels les crimes de violence et plus particulièrement l'homicide, les délits sexuels et surtout l'inceste et le viol, délits contre la propriété, etc. A présent, la victimologie est préoccupée par l'affirmation des droits de la victime et par l'action visant à améliorer son sort. Les progrès théoriques, réalisés grâce aux travaux des pionniers, ont 
été éclipsés par des développements majeurs dans le domaine appliqué mais sont destinés à fournir à la victime les services, l'aide et l'assistance dont elle a besoin et à lui permettre de se rétablir des effets traumatisants de la victimisation.

Plusieurs facteurs ont, à mon avis, contribué à la tendance actuelle. Soulignons les suivants :

1) Un revirement vers la droite dans l'opinion publique et chez ceux qui travaillent dans le système de la justice criminelle. Ce courant n'est pas limité au Canada et aux États-Unis mais s'observe également dans plusieurs autres pays. La position de la droite vis-à-vis le crime et la justice a toujours été très critique à l'égard de la limitation continuelle des peines ou de la notion de réhabilitation et ne cesse d'affirmer que la société favorise les droits et les intérêts des criminels aux dépens des droits et du bien-être de la victime (voir Miller, 1973). Selon cette approche, la responsabilité première de toute société dans le domaine de la justice criminelle consiste à protéger les victimes, et on demande avec insistance qu'on accorde plus d'attention à leurs souffrances et qu'on se soucie davantage de leur sort. Une des conséquences positives de ce revirement vers la droite fut de sensibiliser les professionnels de la justice et le public en général au sort de la victime et aux mauvais traitements que subissent ceux qui sont criminellement victimisés. Plusieurs titres d'ouvrages ou d'articles peuvent être cités afin d'illustrer cette préoccupation grandissante relative au sort de la victime : "Ayons moins de pitié pour le criminel et plus de pitié pour la victime innocente (par D.G. Feeney, 1973), ou «Victimes du crime ou victimes de la justice? (programme subventionné par la Section de la justice criminelle, American Bar Foundation, 1977).

2) Une influence toujours croissante des mouvements de la femme. Dans leur croisade contre le chauvinisme masculin, contre la domination et la suprématie de l'homme, les féministes ont adopté la cause des victimes du viol et celle des femmes battues. Il en est résulté un grand nombre d'ouvrages sur le viol, sur la violence conjugale contre les épouses, traitant les deux sujets dans une perspective féminine. Ces mouvements ont aussi été très influents dans la création, dans plusieurs pays, de centres pour les femmes victimes de violence.

3) Un déclin général de la recherche fondamentale en criminologie par rapport à la recherche appliquée en justice criminelle, favo- 
risée par la disponibilité de fonds considérables en provenance de plusieurs organismes gouvernementaux, dont, en particulier, le LEAA (Law Enforcement Assistance Administration) aux EtatsUnis.

Dans les pages qui suivent, nous allons examiner brièvement quelques tendances et développements récents en victimologie.

Le passage d'une criminologie statique d̀ une victimologie dynamigue : une promesse non remplie

Les débuts de la victimologie, comme ceux de la criminologie, furent foncièrement étiologiques comme on le constate à la lecture, même superficielle, des publications et travaux. C'est ainsi que le premier exposé systématique de victimologie fut celui publié en 1948 par Von Hentig dans son livre Le Criminel et sa Victime. La partie traitant de la victime, à savoir la partie IV du livre, contient un seul chapitre intitulé « $\mathrm{La}$ contribution de la victime à la genèse du crime ». Le but de Von Hentig fut, de toute évidence, de présenter un double cadre pour l'étude du crime et de fournir un substitut et une alternative à l'approche traditionnelle qui était, à toute fin pratique, unidimensionnelle et centrée exclusivement sur l'auteur du délit. Jusqu'à cette époque, toutes les explications de la délinquance tournaient autour des caractéristiques socio-culturelles du criminel, ses anomalies biologiques et/ou ses particularités psychologiques. La plupart des théories, qui tentaient de définir une causalité ou une association, ne fournissaient que des explications partielles et statiques. Cette approche, connue comme l' approche des traits », ignorait complètement, ou minimisait délibérément l'importance des facteurs situationnels qui souvent déclenchent le comportement criminel. L'étude de la victime, de ses caractéristiques, de ses rapports et de ses interactions avec le criminel, de son rôle et de sa contribution à la genèse du crime offrait une perspective nouvelle et une dimension particulièrement prometteuse pouvant transformer l'étiologie criminelle de l'étude statique et unilatérale qu'elle était en une approche situationnelle et dynamique, qui voit le comportement délinquant pas comme une action isolée et bornée mais comme l'aboutissement des processus dynamiques d'interaction :

$\mathrm{Vu}$ que le comportement criminel est un comportement dynamique, il ne peut être expliqué que par une approche dynamique où le délinquant, l'acte et la victime sont des 
éléments inséparables d'une situation totale qui conditionne la dialectique de la conduite anti-sociale (Fattah, 1976).

Les théories criminologiques et les analyses traditionnelles du comportement criminel n'ont jamais réussi à expliquer pourquoi des individus ayant certaines caractéristiques commettent des délits, tandis que d'autres individus ayant les mêmes caractéristiques ne le font pas. En plus, elles n'ont jamais apporté de réponse satisfaisante à la brûlante question, pourquoi le criminel a agi à un moment donné plutôt qu'à un autre, ou pourquoi il a commis son crime dans telle situation plutôt que telle autre et contre une victime particulière ? La victimologie semblait offrir la possibilité d'intégrer les facteurs prédisposants aux facteurs actualisants, les variables individuelles aux variables situationnelles. $\AA$ travers la victimologie, il apparaissait possible de développer un modèle dynamique du comportement délictuel incorporant les motifs de l'auteur et l'attitude de la victime, l'initiative de l'agresseur et la réponse de l'agressé, l'action du prédateur et la réaction de la proie. Cette promesse reste cependant largement non remplie.

\section{Le concept de «victime catalyseuse : une critique injustifiée}

Les efforts visant à jeter la lumière sur le rôle effectif de la victime dans la genèse du crime et à établir si la victime a instigué, initié, provoqué, favorisé ou facilité le délit, ainsi que les tentatives de développer un modèle dynamique qui déborde l'approche des traits et des attributs, ont subi un recul et ont été sapés par une critique véhémente du concept de la victime catalyseuse. Les attaques souvent injudicieuses et injustifiées, venaient de plusieurs quartiers. Les plus féroces sont probablement celles des groupes féministes, particulièrement exaspérés par le taux élevé d'acquittements, dans les cas de viol, et par les traitements infligés aux victimes de ce crime par le système de la justice criminelle.

Une bonne partie de la critique fut adressée non pas au concept lui-même, mais à la façon dont il a été opérationnalisé, non pas à la notion selon laquelle certaines victimes peuvent, dans certains cas, provoquer, précipiter, ou contribuer et participer au crime, mais à la définition donnée au concept dans quelques études victimologiques et, plus particulièrement, dans l'étude d'Amir sur le viol à Philadelphie. La position prise par Clark et Lewis est typique de la critique faite de l'étude d'Amir. Dans leur 
livre Le Prix de la sexualité coercitive, publié en 1977, les auteurs écrivent :

Amir's unquestioning acceptance of the male perspective is not unique ; it is a widespread feature of male-dominated Society. But when this general bias is carried into the social sciences, it becomes an academic endorsement of the rapist's point of view, and an excuse for blaming rape upon its victims (p. 153).

Malheureusement, certains auteurs ont étendu leurs critiques du concept de la victime catalyseuse à la victimologie tout entière l'accusant d'être "l'art de blâmer la victime»; de tenter de disculper le criminel et d'inculper la victime (voir par exemple, chapitre X du livre de Clark et Lewis, 1977). Les citations qui suivent, tirées de ce livre sont de bons exemples de la campagne menée par certains féministes contre la jeune discipline de la victimologie :

In the social sciences, victim blaming is becoming an increasingly popular rationalization for criminal and deviant behavior... over the past few years, victim blaming has become institutionalized within the academic world under the guise of victimology (p. 147).

The male researcher finds his escape in victimology. He seeks the problem's cause in the behavior of its victim, and goes on to persuade himself and the public at large that by changing that behavior, the problem can be controlled. In this way, the study of victimology becomes the art of victim blaming (p. 150).

Les critiques du concept de la victime catalyseuse, hommes, ou femmes, ne semblent pas réaliser qu'il y a une distinction subtile qu'il faut faire entre le concept comportemental (behavioristic concept) utilisé dans les études étiologiques afin d'expliquer la genèse du crime et le concept légal (tel que la provocation) utilisé dans les procès criminels pour trancher la question de culpabilité et pour choisir la sanction la plus appropriée. L'emploi fréquent dans le langage victimologique des termes empruntés à la nomenclature légale tels que «culpabilité », " responsabilité », "blâme », etc. a beaucoup contribué à la confusion. Et des titres tels que Nicht der Mörder, der Ermordete ist Schuldig (Franz Werfel, 1920) ; The murdered one, too is guilty (Lydia Sicher, 1936) ; et La Victime est-elle coupable? ont fourni des munitions additionnelles aux critiques. 
Malgré les attaques, il n'en reste pas moins que le concept de la victime catalyseuse, tel qu'originalement énoncé par Marvin Wolfgang dans son étude sur l'homicide à Philadelphie en 1958, est fondamentalement valable. Particulièrement dans les cas, où le crime fut l'aboutissement d'une longue ou brève interaction entre l'agresseur et la victime, il est pratiquement impossible d'expliquer la genèse du comportement de l'auteur ou de comprendre ses motifs, sans examiner la psychodynamique des deux protagonistes et sans analyser la socio-dynamique de la situation. Ce processus implique, inéluctablement, l'étude de l'attitude et du comportement de la victime ainsi que celle de la perception, de l'interprétation, et de la réaction que peut en avoir le criminel. Contrairement aux concepts statiques de vulnérabilité ou de prédisposition, le concept de la victime catalyseuse, est un concept dynamique qui permet une meilleure compréhension non seulement du \& pourquoi > du crime, mais aussi du pourquoi il fut commis dans une situation donnée contre une victime en particulier. Que le concept ait été défini assez largement ou opérationnalisé assez libéralement dans une ou plusieurs études n'est pas une raison pour le rejeter complètement, ni pour nier sa valeur explicative, sa validité et sa contribution potentielle à l'étiologie du comportement criminel. Personne ne contestera, par exemple, la définition que Wolfgang (1958) a donnée dans son étude sur l'homicide de la victime catalyseuse :

The term victim-precipitated is applied to those criminal homicides in which the victim is a direct, positive precipitator in the crime. The role of the victim is characterized by his having been the first in the homicide drama to use physical force directed against his subsequent slayer. The victim-precipitated cases are those in which the victim was the first to show and use a deadly weapon, to strike a blow in an altercation - in short, the first to commence the interplay of resort to physical violence (p. 252).

Les cas cités par Wolfgang pour illustrer ce qu'il entend par "l'homicide catalysé par la victime » ne laissent aucun doute quant à la justesse du concept et la façon dont Wolfgang l'a opérationnalisé.

Dans son étude sur le viol, Amir $(1967,1971)$ a utilisé une définition assez différente et beaucoup plus large que celle élaborée par Wolfgang pour l'homicide. Vu la délicatesse du sujet et l'ambiguité de la définition, son étude fut fortement critiquée. 
Clark et Lewis (1977) prétendent que Menahim Amir a confondu le comportement de la victime du viol avec l'interprétation que le criminel donnait à ce comportement (p. 154). Ils lui reprochent de n'être jamais arrivé à la conclusion logique, à savoir que * c'est l'interprétation erronée du criminel qui précipite le viol . Évidemment, Clark et Lewis ne réalisent pas que la perception propre du délinquant (qu'il soit un tueur, violateur, fraudeur ou autre) de l'attitude de la victime et son interprétation subjective du comportement de cette dernière (si erronée que cette interprétation puisse être) sont extrêmement importantes pour la compréhension de sa personnalité, de ses motifs et de son crime, même si elles ne constituent pas de raisons valables pour réduire les accusations ou pour mitiger la sanction. Que la perception ait été exacte ou inexacte, que l'interprétation du comportement de la victime ait été correcte ou incorrecte, que le délinquant ait été justifié ou non de conclure ce qu'il a conclu, et d'agir comme il a agi, sont toutes des questions que les cours criminelles et les panels de dédommagement doivent trancher. Les experts en sciences sociales, sont intéressés par l'explication, et non par la justification. Ils veulent comprendre le comportement et non pas le rationaliser. Ils sont préoccupés par l'étiologie et non pas par les excuses légales et les circonstances atténuantes. L'attribution d'une faute à la victime (qu'elle soit victime du viol ou de n'importe quel autre crime) est sans doute une des techniques que les avocats de la défense utilisent très fréquemment pour assurer l'acquittement de leur client, ou pour lui obtenir un traitement plus indulgent. Par contre, les criminologues font face à un autre problème lorsqu'ils tentent d'expliquer le crime. Ils doivent pouvoir discerner les perceptions réelles du délinquant (même si elles sont erronées) et ses interprétations sincères du comportement de la victime (même si elles ne sont pas exactes), ainsi que les rationalisations ex post facto, souvent exprimées par les délinquants pour justifier leur conduite.

Les critiques de la victimologie ne semblent pas se rendre compte que des concepts explicatifs tels que le concept de la victime catalyseuse ou de criminalité provoquée par la victime, n'impliquent pas l'imputation d'une faute ou l'attribution du blâme à la victime. Ils ne font que référer au rôle actuel ou potentiel que l'attitude et le comportement de la victime ont pu jouer ou pourraient jouer dans la genèse du crime. 
Faire allusion à la négligence de la victime, à sa nonchalance, à son imprudence ou à son manque de précaution est tout simplement une façon, parmi tant d'autres, de mettre en évidence l'importance des facteurs situationnels ou actualisants et de souligner la relation étroite entre certains délits et les opportunités de les commettre. De même, quand par exemple dans un cas de parricide on fait référence aux mauvais traitements que le fils subissait coitinuellement de la part de son père, cela ne devrait pas être interprété comme un effort délibéré de blâmer la victime ou de disculper l'assassin. C'est une tentative d'expliquer les motifs de l'agression et d'analyser la chaîne d'événements qui a finalement abouti à la commission du crime.

En mettant l'accent sur l'instigation ou la contribution éventuelle de la victime à certains crimes, les victimologues ne font qu'attirer l'attention sur le conflit qui parfois existe entre la fonction protectrice de la loi et le fait que certaines victimes ne veulent pas être protégées. Nulfe part, ce conffit n'est plus évident que dans le viol statutaire ou dans les cas de lois interdisant la vente des boissons alcooliques ou du matériel pornographique aux mineurs, ainsi que l'accès à des endroits spécifiques tels que les casinos de jeu ou les pistes de courses. En soulignant l'échec ou le refus de la victime de se protéger ou de protéger ses biens, les victimologues ne font que mettre en lumière le fait que certaines victimes ne veulent pas se prévaloir des moyens de protection mis à leur disposition. N'est-il pas paradoxal qu'on se trouve obligé de forcer les chauffeurs d'automobiles de boucler leurs ceintures par le truchement de sanctions criminelles?

\section{L'attitude du criminel et de la victime}

Les débuts de la victimologie furent caractérisés par une préoccupation presque exclusive concernant les attributs des victimes de certains crimes, le rôle causal de la victime et les modèles des rapports entre les criminels et les victimes. Cependant, à l'exception de quelques cas où la victime fut un catalyseur direct du crime, le fait de découvrir une relation primaire ou secondaire entre le criminel et sa victime est très souvent sans grande valeur dans l'explication de la motivation ou des activités des deux parties. La victimologie actuelle a étendu la perspective inter- 
personnelle d'une simple typologie des relations pour englober une étude approfondie des attitudes réciproques du criminel et de la victime et de leurs perceptions l'un de l'autre. $\AA$ notre avis, cela constitue un des développements théoriques les plus importants des dernières années. Non seulement a-t-il ouvert des horizons nouveaux à l'étude des interactions délinquant-victime, mais il a aussi offert une voie nouvelle à la compréhension du dynamisme du comportement criminel et du passage à l'acte. En outre, la recherche dans ce domaine aura certainement des applications importantes dans les champs de la prévention, de la réhabilitation, de l'éducation et du traitement de la victime.

\section{L'attitude du criminel à l'égard de la victime}

Plusieurs criminologues, tels Sutherland (1937), Redl et Weinman (1951), Cressey (1953), Sykes et Matza (1957) ont, dans le passé, parlé de l'attitude qu'a le criminel envers sa victime et ont attiré l'attention sur le rôle important de cette attitude dans les processus de sélection de la victime et de rationalisation de l'acte. Récemment, la littérature sur le viol a mis l'accent sur l'influence qu'exerce la culture sur les perceptions que le délinquant a de sa victime. Les études victimologiques semblent indiquer que toute société en stigmatisant certains individus ou certains groupes rend légitime leur victimisation et les désigne implicitement ou explicitement comme des cibles appropriées aux attaques criminelles.

\section{Le processus de désensibilisation}

La plupart des délits tels que l'homicide, les voies de fait, le viol, le vol et la fraude, ne sont que quelques exemples qui causent des dommages physiques et/ou matériels à la victime. Souvent, ils impliquent le fait d'infliger des souffrances à un être humain. Or, tous les criminels ne sont pas totalement dépourvus de sensibilité, de sentiments de pitié ou d'empathie. Leur crime peut donc entraîner chez eux une crise morale. Afin d'éviter cette tension morale, le sentiment de culpabilité et les remords qui peuvent y être associés, les criminels ont d'habitude besoin de se désensibiliser à l'avance à l'égard des douleurs et des souffrances de la victime. Cette désensibilisation sert également à neutraliser la résistance morale, à surmonter les inhibitions et à réduire au silence la conscience du délinquant. La légitimation de l'acte, la 
négation ou la réification de la victime, la dérogation et la dévalorisation de celle-ci, sont des mécanismes importants dans le processus de désensibilisation. En même temps que fut élaboré par les criminologues le paradigme théorique de l'utilisation de la victime comme agent de légitimation de soi, des études du même processus ont été menées indépendamment par des psychologues sociaux. Celles-ci tendent à confirmer que la désindividualisation et la dérogation de la victime sont aptes à désinhiber le délinquant potentiel et à faciliter le passage à l'acte. Les études psychologiques ont démontré que lorsque la victime n'est pas considérée comme un individu, un état de désindividualisation peut en résulter et mener à l'affaiblissement des contraintes internes et des mécanismes de contrôle. Elles ont également démontré que la dérogation et la dénigration de la victime conduisent à un sens amoindri de responsabilité et à une dissonance réduite à la suite de l'agression.

Les explications psychologiques du processus de dérogation/ dénigration sont largement inspirées par l'hypothèse du * monde juste » énoncée par Lerner et supposent ainsi que les gens ont besoin d'y croire. Mais il nous semble que le processus est plutôt une indication de l'effort que fait le criminel pour se désensibiliser, pour justifier son comportement dommageable et pour rationaliser ses actions injurieuses. Ceci semble être confirmé par les études de Cialdini et al. (1975), selon lesquelles l'élément médiateur dans le phénomène de dérogation de la victime n'est pas la tendance à croire en un juste monde, mais la tendance à justifier sa complicité dans un acte qui cause du mal à autrui. En tout cas, les implications de ces études psychologiques pour la victimologie sont évidentes. Une analyse approfondie du processus de désensibilisation est apte à jeter de la lumière sur les forces qui transforment un individu apparemment normal et bien socialisé en une personne agressive et sans inhibitions, capable de commettre des actes d'une extrême cruauté et même de sauvagerie en contradiction avec son propre système de valeurs; sans la moindre trace de compassion, d'empathie ou de pitié pour la victime et sans éprouver des remords ou des sentiments de culpabilité. D'autre part, en rendant le délinquant plus conscient et plus sensible aux souffrances de la victime (et la restitution est sûrement un des moyens par lesquels ce but pourrait être atteint), il serait possible de développer des techniques efficaces de réhabilitation et de prévention. 
Les images stéréotypes que les criminels ont des victimes

Malgré la pénurie d'études sur ce sujet, il semble exister chez les délinquants des définitions stéréotypées et des images consensuellement vérifiées des victimes. Il y a quelques années, Hermann et Julia Schwendinger (1967) ont entrepris une étude des stéréotypes des victimes. Dès le début, ils étaient capables d'observer ce qui semblait être une entente tacite parmi les délinquants, que la victime est un être humain sans valeur. Leurs données ont renforcé l'hypothèse que les délinquants maintiennent tacitement une attitude commune envers la victime.

De même, dans son étude du voleur professionnel, Sutherland (1937) donne une bonne description des images stéréotypées que les voleurs professionnels ont de leurs victimes potentielles. Ils les désignent comme étant des :

... suckers who are golating over their prospective gain... with larceny in their souls... and with little sympathy for those they are hoping to beat.

Les chercheurs qui ont étudié le viol insistent sur le fait que les auteurs ont une image dévalorisée de la femme. Ils la regardent comme un objet sexuel, une pièce de propriété qui doit consentir, se soumettre et souffrir ${ }^{1}$. Les conducteurs mâles semblent également entretenir des stéréotypes en ce qui a trait aux filles qui « font du pouce ». Ils les voient comme désireuses et/ou consentantes ou, au moins, comme des filles prêtes à prendre le risque d'être violées. Ceci influence les interactions entre ces conducteurs et les filles qu'ils acceptent de transporter et déforme largement leurs perceptions de ce qui s'est passé. Dans leur esprit, il n'y a pas eu de crime. Ce n'était pas un viol, mais tout simplement un plaisir sexuel partagé.

Les études comparatives du type transculturel pourront être extrêmement utiles si on $y$ examine, analyse et compare les stéréotypes et les images que les délinquants entretiennent de leurs victimes. Elles nous aideront à comprendre comment et de quelle façon les interprétations culturelles de l'attitude et du comportement de la victime contribuent à motiver le délinquant, influencent son choix d'une cible particulière et l'aident à rationaliser ou à justifier son délit.

1. Voir Robert, P. et al. (1977), L'lmage du viol collectif, Genève. 


\section{Les perceptions qu'ont les criminels des victimes méritoires}

Les études victimologiques indiquent que les délinquants, en général, font des distinctions subtiles entre des cibles appropriées et des cibles inappropriées à la victimisation. Ils semblent dresser une ligne de démarcation assez nette entre ceux qui peuvent et ceux qui ne peuvent pas être victimisés (voir, par exemple, Sutherland, 1937 ; Thrasher, 1947 ; Sykes et Matza, 1957 ; Dynes et Ouarantelli, 1970). Deux catégories apparaissent comme particulièrement vulnérables à la victimisation : la victime coupable et la victime culturellement légitime.

La victime coupable - L'imputation de culpabilité à posteriori à la victime n'est pas un phénomène nouveau. Le langage populaire est plein de dictons (tels que «he has himself to blame », «he had it coming to him ou 《he asked for it $\gg$ ) qui sont utilisés pour indiquer que la victime était au moins partiellement responsable de ce qui lui est arrivé. Mais c'est à la victimologie que revient le mérite d'avoir attiré l'attention sur le processus mental de rationalisation dans lequel le fait de blâmer la victime est utilisé comme une technique efficace de neutralisation. Ce processus diffère des justifications ex post facto car il précède plutôt que ne succède à la commission du délit permettant ainsi au délinquant de surmonter ses inhibitions, de faire taire sa conscience et d'éviter d'avance tout sentiment de culpabilité, honte ou remords qui pourrait intervenir après le délit.

L'antériorité de la culpabilité de la victime (que cette culpabilité soit réelle ou imaginée par le délinquant, qu'elle soit engendrée par des actions de la victime ou par simple association) semble débarrasser le délinquant potentiel presque complètement de tout sens de culpabilité et fournir à sa conscience un alibi parfait. Elle lui permet de légitimer son délit, de redéfinir son agression comme un acte de justice vengeresse, de nier sa propre culpabilité et de mettre le blâme sur le dos de la victime. Blâmer la victime, même dans les cas où la victime est entièrement innocente, n'est pas un processus de déformation consciente et intentionnelle. Dans beaucoup de cas, le délinquant est profondément convaincu de la culpabilité de sa victime. Ceci est vrai dans un grand nombre de crimes passionnels aussi bien que dans les délits des paranoïaques. En effet, le crime passionnel est presque invariablement caractérisé par une attitude justiciaire de la part du délinquant, une attitude qui est une des motivations 
conscientes et justificatrices du crime ${ }^{2}$. Dans tous les crimes motivés par la vengeance et dans beaucoup de délits politiques également, le fait injurieux est vu, vécu et perpétré en tant qu'un acte de justice, une manifestation de rétribution justifiée qui s'impose. La technique de blâmer la victime est aussi évidente dans le génocide. Ici, l'extermination de toute une minorité raciale ou religieuse est tenue non seulement comme un fait utile et désirable mais aussi comme nécessaire et justifié. Précédant les actes de génocide, le groupe minoritaire est blâmé pour toutes sortes de maux sociaux et économiques de façon à éliminer toute sympathie ou compassion à son égard et afin que son anéantissement apparaisse comme une fin juste et légitime.

Mais le processus de blâmer la victime n'est pas limité aux crimes de violence ou aux délits contre la personne. Souvent le fait de frauder le fisc est vu comme représaille contre un gouvernement qui impose des impôts trop élevés ou inéquitables. Même la trahison peut être légitimée et rationalisée en tant qu'un acte de vengeance contre un régime injuste, oppressif et autocratique.

La victime culturellement légitime - Le concept de « la victime légitime $\gg$ est une autre contribution récente de la victimologie. Il fut utilisé, la première fois, en 1973, par Weiss et Borges pour décrire la victime du viol. Ces auteurs notent que la socialisation (et plus particulièrement l'apprentissage des rôles sexuels) exploite les deux sexes et produit ainsi des délinquants et des victimes légitimes. A leur avis, une société dominée par les mâles, où toutes les positions de pouvoir et d'influence sont occupées par les hommes, tend à établir et perpétuer la femme comme cible légitime de victimisation.

Social processes prepare the woman for her role as a potential victim and provide the procedures to make her a socially approved or legitimate victim for rape (Weiss et Borges, 1973).

Dans les sociétés primitives, chez les bandes d'adolescents et parmi les groupes politiques extrémistes, les actes de violence contre les membres du groupe externe (out-group) ne sont pas seulement tolérés mais sont quelquefois encouragés tandis que toute violence contre les membres du groupe interne (in-group) est fortement condamnée. Le système normatif de ces sociétés et groupes désigne les membres des groupes externes (out-groups)

2. Voir Hesnard, A. (1951), La Psychologie du crime, Paris, Payot. 
comme des cibles légitimes et appropriées aux attaques violentes et agressives ${ }^{8}$. On peut donc affirmer que dans toute société il existe des victimes légitimes, c'est-à-dire des personnes ou des groupes contre qui l'emploi de la violence est encouragé, toléré ou approuvé. À titre d'exemple on peut citer les individus qui occupent des rôles sociaux dévalorisés et qui serviront toujours de cibles populaires aux agressions. Une enquête nationale sur les attitudes du peuple américain vis-à-vis la violence a conclu que excluding people from groups to which one feels related can serve as a rationalization justifying violence toward them... On peut donc rendre la violence infligée à ces individus plus facilement acceptable (voir Blumenthal et al., 1972 ; Conklin, 1975).

L'emploi de la courroie dans les écoles pour punir les enfants, de la violence dans les institutions pénales pour discipliner et contrôler le comportement des détenus, la flagellation des délinquants coupables de certains crimes ; sont considérés presque universellement comme des formes légitimes de violence et ceux à qui ces peines sont infligées sont regardés comme des cibles appropriées et méritoires.

Dans plusieurs juridictions le fait de consommer l'acte sexuel par force avec sa propre épouse ne constitue pas un délit criminel et n'est pas qualifié de viol. Donc si l'épouse est violée par son mari, elle est une victime légitime. Le concept s'étend à d'autres formes de violence à l'intérieur de la famille. Les enfants sont des victimes légitimes lorsque les parents utilisent la force physique et les punitions corporelles au cours de l'éducation ou de l'apprentissage. Et pendant très longtemps la violence entre mari et femme fut considérée comme légitime, et par la police, et par les tribunaux. De même, dans plusieurs pays l'épouse infidèle est l'exemple typique de la victime légitime. Dans ces pays, le mari qui tue sa femme adultère (surtout s'il la surprend en flagrant délit) est dispensé de toute peine ou est traité avec une extrême indulgence.

\section{Proximité et distance, rapprochement et éloignement de la victime}

Les criminologues savaient depuis quelque temps que pour passer à l'acte il est souvent nécessaire pour le criminel de réduire la distance physique et d'élargir la distance affective entre lui et sa victime. La commission d'un délit contre une victime imperson-

3. Voir Robert, P., Les Bandes d'adolescents, Paris. 
nelle ou intangible (tel le gouvernement ou une corporation) évoque moins de résistance morale et exige moins de courage psychologique qu'une attaque dirigée contre un être proche, perceptible et touchable. Plus grande est la distance physique moins conscient est le délinquant de la souffrance de sa victime. Un écart assez large rend la tâche de causer du mal à la victime moins difficile et moins chargée de tension morale. Ainsi, le fait de bombarder des cibles lointaines éveille moins d'inhibitions que le fait de poignarder ou de fusiller des femmes et des enfants face à face ou à courte distance.

Les études de laboratoire menées par des psychologues sociaux ont abouti à des conclusions à la fois importantes et intéressantes. Ainsi dans ses expériences bien connues, Milgram (1974) a examiné l'effet de la proximité physique de la victime sur des sujets chargés de lui administrer des chocs électriques. Milgram voulait vérifier l'hypothèse que plus on approche la victime du sujet (c'est-à-dire en la rendant plus saillante, plus visible et en augmentant ainsi la conscience du sujet de ses souffrances et de sa détresse), plus la performance du sujet sera réglementée et plus sera réduite son obéissance à celui qui conduit l'expérience. Les résultats de l'expérience confirmaient l'hypothèse et révélaient que l'obéissance au conducteur fut réduite de façon significative lorsqu'on a placé la victime dans la proximité immédiate du sujet. Ainsi, $35 \%$ seulement des sujets ont défié le conducteur de l'expérience dans les conditions de distance comparé à $37,5 \%$ dans les situations de "voice feedback 》, à $60 \%$ dans les conditions de proximité et à $70 \%$ dans la situation où la victime fut si proche qu'elle pût être touchée. En commentant ses résultats, Milgram note que dans la situation où la victime est lointaine, ses souffrances ont un caractère plutôt abstrait et le sujet peut les écarter. Bien sûr, il est conscient, mais seulement dans un sens conceptuel, que ses actes causent de la douleur à une autrc personne. Ce fait est craint mais pas ressenti. La distance permet de rétrécir le champ cognitif à un stade où la victime est éliminée de l'esprit du sujet. Par contre, quand la victime est proche, il est plus difficile de l'exclure de la pensée. Dans les conditions de proximité, le sujet peut sentir qu'il est devenu plus saillant dans la sphère de conscience de la victime et, par conséquent, il devient plus conscient de soi, plus embarrassé et plus inhibé dans sa punition de la victime (Milgram, 1974, p. 32-43). 
Une empathie pour la victime et une conscience plus accentuée de ses souffrances, constituent, de toute évidence, des forces inhibitrices et contrôleuses. Malheureusement, la plupart du temps elles ne sont pas suffisantes pour prévenir le crime. Autrement, comment peut-on expliquer le fait qu'une proportion très élevée de tous les homicides et autres délits de violence est commise contre les individus les plus proches physiquement et affectivement, des agresseurs?

\section{L'attitude de la victime à l'égard du délinquant}

Plusieurs délits sont commis à l'insu de la victime et sans aucune confrontation directe avec le délinquant. Dans de tels cas, on peut difficilement parler d'une attitude spécifique de la victime envers le malfaiteur. Plus intéressants sont donc les cas où une interaction ou quelques agissements personnels eurent lieu entre le criminel et la victime. La recherche dans ce domaine est encore à son enfance. Quelques études sur les survivants des camps de concentration nazis ont examiné entre autres les attitudes des victimes vis-à-vis leurs bourreaux. Récemment on constate un intérêt renouvelé pour ce sujet dû aux observations faites auprès des victimes de certains actes terroristes. On a constaté, en effet, que dans certains cas d'enlèvement, de prise d'otages, de piraterie aérienne, surtout si l'épreuve a duré plusieurs jours ou semaines, quelques victimes réagissent par une réponse émotionnelle positive plutôt que négative à l'égard des agresseurs. Cette réaction initiale pourrait même se développer et devenir un attachement émotif profond ou un lien affectif. Ce phénomène curieux a suscité plusieurs explications. Certains auteurs sont d'avis que de tels liens affectifs positifs résultent de l'état de dépendance qui se développe entre l'otage et son ravisseur. D'autres auteurs expliquent le phénomène en se référant au concept freudien de l'« identification avec l'agresseur ». Un troisième point de vue souligne l'importance de forts sentiments de gratitude qu'éprouve le survivant à l'égard de son ravisseur pour avoir épargné sa vie.

Most experiences, particularly those of a prolonged character, reliably and the development of an empathy between the terrorist and the hostage victim. It is suggested by some that, in prolonged cases, a state of dependency develops between the hostage and his keeper, to the extent even of giving rise to serious ethical and identity problems on the part of the victim (H.H.A. Cooper, 1976, p. 236). 


\section{Le syndrome de Stockholm}

Il s'agit d'un ré-alignement d'affection, dramatique et inattendu, qui se transforme en un lien positif entre l'otage et le ravisseur, souvent accompagné d'une certaine hostilité et méfiance chez la victime à l'égard des autorités. Le terme fut forgé à la suite d'un incident célèbre qui eut lieu en Suède en 1973. Après avoir commis un vol à main armée dans la Kreditbank, à Stockholm, les deux bandits prirent quelques employés comme otages et les gardèrent dans la voûte de la banque du 23 au 28 août. Par la suite, on a décrit en détail le développement d'un lien affectif entre une femme otage et l'un des voleurs. Après leur libération, les victimes ont demandé que les malfaiteurs soient traités avec indulgence.

Quoique le syndrome de Stockholm ne se développe pas dans tous les cas d'enlèvement ou de prises d'otages il ne semble pas être très rare. Des réactions similaires à celles observées chez les victimes de l'incident de la banque suédoise, avec ou sans rapports sexuels entre l'otage et le ravisseur, ont été rapportées dans plusieurs autres cas d'enlèvement et de siège et ont reçu une énorme publicité dans le cas de l'héritière américaine : Patricia Hearst.

Ochbert (1977) note que les liens affectifs positifs ne s'installent pas immédiatement quoiqu'ils semblent bien établis au troisième jour de l'épreuve. Il souligne trois facteurs qui, à son avis, semblent favoriser le développement du syndrome de Stockholm :

a) l'intensité de l'expérience ;

b) la durée de l'épreuve (bien qu'après trois ou quatre jours, la durée ne semble pas être tellement importante);

c) la dépendance de l'otage de son bandit pour survivre, et la distance psychologique qui sépare l'otage des autorités.

On a aussi observé un syndrome similaire chez les passagers de trains ou d'avions détournés. Au lieu de réagir à leur victimisation avec rage, colère et indignation, quelques passagers semblent émerger de leur expérience traumatisante plein de louanges pour les pirates. Les études systématiques de ce phénomène sont assez rares et il n'est pas surprenant qu'on ne soit pas encore arrivé à une explication satisfaisante. Le Dr Hubbard, auteur d'un 
livre sur les pirates de l'air, a déclaré dans une entrevue avec Time Magazine (oct. 1976) que les pirates bénéficient habituellement d'une hostilité assez répandue envers l'autorité. Une fois que le passager est convaincu qu'il ne sera pas tué, il tend à regarder le pirate comme un desperado qui s'en prend aux pouvoirs en place. Hubbard ajoute qu'il n'est pas rare que les victimes voient l'acte de détournement comme un billet gratuit pour l'aventure et la publicité. En outre, vu que le pirate n'utilise pas toute la force dont il dispose, les passagers se sentent reconnaissants.

Une opinion similaire fut exprimée par le psycho-linguiste Murray Miron qui note que le pirate gagne l'admiration en menaçant. "Quelqu'un qui tient votre vie dans ses mains vous récompense chaque fois qu'il ne vous tue pas. Même un geste banal tel que de permettre aux passagers d'allumer une cigarette ou d'utiliser la salle de bain agit comme un lien subtil au ravisseur. *

Selon le psychiatre américain Lawrence Freedman, le lien qui s'établit entre le ravisseur et l'otage, une alliance qui les unit dans leur condamnation de l'intransigeance des autorités et le fait qu'ils déplorent le refus de celles-ci de capituler à leurs demandes, est tout simplement une manifestation de ce que les freudiens appellent l'《identification avec l'agresseur ». Par cela, ils réfèrent à l'identification de l'enfant à une image parentale punitive et à son incorporation des qualités agressives de ce même parent. Cette explication cependant, comme Ochberg (1977) le fait remarquer, ignore le fait que les victimes n'incorporent pas nécessairement la violence des terroristes. Ochberg tend plutôt vers une explication basée sur l'hypothèse du « lien de reconnaissance ». Il écrit :

It seems rather that hostages successfully deny the danger engincered by the terrorists. Having separated this from awareness, they are overwhelmingly grateful to the terrorist for giving them life. They focus on the captor's kindnesses, and not his acts of brutality. Intellectual appreciation of the terrorists' cause may be related to this irrational affection, but the relationship is not complete. That is, one can love a captor and not his cause, and vice versa.

De la responsabilité fonctionnelle de la victime à la responsabilité sociale envers la victime

La victimologie fut accusée, à tort ou à raison, de blâmer la victime, d'insister trop sur son rôle et d'avoir exagéré sa contri- 
bution à la genèse du crime. Les victimologues furent accusés de vouloir disculper le criminel et inculper la victime. $\AA$ cause de cela, peut-être, la victimologie actuelle est très soucieuse de réaffirmer la responsabilité qu'a toute société envers les victimes du crime, ainsi que l'obligation sociale de redresser le tort subi et d'offrir aux victimes une aide et une assistance adéquates.

II y a environ dix ans, et plus précisément en 1968, Stephen Schafer, un des pionniers de la victimologie, publia une monographie pour laquelle il a intentionnellement choisi le titre inverse de celui de Von Hentig publié vingt ans plus tôt. Dans son livre intitulé La Victime et son criminel : une étude de responsabilité fonctionnelle, Schafer parle de la responsabilité de la victime dans sa propre victimisation et de son obligation de faire tout son possible pour prévenir le crime. La façon dont Schafer traite le concept de la responsabilité fonctionnelle de la victime laisse beaucoup à désirer, puisqu'il n'y consacre que les deux dernières pages de son ouvrage. Malgré cette insuffisance, la citation suivante explique bien ce que Schafer entend par ce concept :

Also, it is far from true that all crimes « happen s to be committed ; often the victim's negligence, precipitative action, or provocation contribute to the genesis or performance of a crime. The norm-delineated functional role of the victim is to do nothing to provoke others from attempting to injure his ability to play his role. At the same time, it expects him actively to prevent such attempts. This is the victim's functional responsibility (p. 152).

Paradoxalement, le livre de Schafer contient un plaidoyer fort et passionné pour une reprise de conscience de l'importance de la victime et des demandes pressantes pour des systèmes adéquats de restitution et de dédommagement aux victimes.

Dans le climat qui règne actuellement au sein du système de la justice criminelle et vu l'attitude punitive qu'on constate chez le public en général, parler de la responsabilité fonctionnelle de la victime équivaut à l'hérésie et ne peut susciter que des attaques et des critiques. Par contre, l'appel de Schafer et de tant d'autres pour des systèmes étatiques de compensation et d'indemnité aux victimes a été suivi par presque toutes les sociétés occidentales. En tout cas, le souci actuel pour ceux qui subissent les conséquences des crimes inaugure un deuxième âge d'or de la victimologie. La question posée par les organisateurs de la conférence annuelle de 
l'Association đu Barreau américain (ABA), section de la justice criminelle (Chicago, le 10 août 1977), illustre bien cette nouvelle prise de conscience du sort et des besoins de la victime. De même, dans son introduction aux Actes de la réunion, B. James George, Jr., pose quelques questions pertinentes et d'actualité. Il se demande * Are the victims of crime also the victims of the criminal justice itself ? Does the system give the victim any rights... while it religiously keeps watch to preserve the rights of the defendant? Should victims of crime be eligible to receive compensation from the State, restitution from the offender, or civil damages from third parties?

Ces mêmes questions furent posées deux années plus tôt à la Conférence internationale de victimologie tenue à Bellagio, en juillet 1975. La section traitant de «la victime et le système de la justice criminelle fit plusieurs recommandations qui, à toutes fins pratiques, constituent une charte des droits des victimes. Non seulement le groupe affirma le droit de la victime à la restitution et à la compensation, mais il insista aussi sur le besoin qu'a la victime d'être traitée équitablement et d'une façon humaine par le système de la justice criminelle et par la société tout entière. Ceci signale l'entrée de la victimologie dans une ère nouvelle : la redécouverte de la victime.

\section{Effets des caractéristiques de la victime} sur les décisions de la justice criminelle

Jusqu'à très récemment, peu de recherches furent entreprises pour déterminer si la victime exerce une influence quelconque sur le processus de la justice criminelle et sur l'issue finale de son procès. En accord avec le souci général manifesté ces dernières années à l'égard des victimes, on peut constater un intérêt croissant portant sur le rôle que pourraient jouer les caractéristiques et le comportement de la victime. Cet intérêt a suscité un certain nombre d'études empiriques sur le sujet. Ainsi les études expérimentales, menées par les psychologues, ont démontré que les observateurs sont aptes à être influencés, et dans la détermination de culpabilité, et dans leur choix de sentences, par les caractéristiques de la victime. Malgré ces études expérimentales, on se trouve toujours face à une pénurie de recherches basées sur des cas réels et visant à établir l'impact des caractéristiques et du comportement de la victime sur les décisions de la justice. 
Quoi qu'il en soit, c'est un fait bien connu que la victime joue un rôle primordial de par sa décision de signaler ou de ne pas signaler le délit. Cette prise de position initiale est décisive puisque dans la majorité des cas la mobilisation de la justice est subordonnée à la plainte de la victime. Ceci est confirmé par des études sur les activités policières dans quelques villes américaines qui montrent que la grande partie du travail policier (plus des trois quarts) est initiée par les citoyens et qu'un pourcentage mineur est initié par la police elle-même (voir Black, 1970). Une fois que la victime a porté plainte, les décisions subséquentes aux différentes étapes du processus semblent être considérablement influencées par les caractéristiques et la conduite de la victime. Les quelques études disponibles, comme celles de Williams (1967a, et $1976 \mathrm{~b}$ ), semblent indiquer que la décision de la police de procéder (acte criminel, déclaration sommaire de culpabilité, etc.) ainsi que l'issue finale du procès sont influencées largement ou en partie par la victime. Ces études révèlent également que les désirs et les attentes des victimes sont généralement considérés et honorés et par la police, et par le procureur, sauf dans les cas où il s'agit d'un crime grave et où la victime ne veut pas procéder formellement en présentant la plainte.

Il va sans dire que la provocation de la victime a toujours été l'un des facteurs qui influencent la décision de la Cour dans les affaires criminelles. Dans certaines juridictions, la provocation constitue une excuse légale, dans d'autres, une circonstance atténuante. Et même dans les cas où les agissements de la victime n'atteignent pas la gravité exigée par la loi et ne répondent pas à la définition légale de «provocation 》 de tels agissements mènent d'habitude à une accusation moins lourde, à une sentence réduite ou même à un verdict de non-culpabilité. Wolfgang (1958) a constaté lors de son étude de l'homicide en Philadelphie que :

A significantly smaller proportion of offenders in victimprecipitated homicide (62\%) than in non-victim-precipitated homicide $(82 \%)$ were found guilty (p. 300$)$.

Indépendamment de la provocation et de la décision d'accepter ou de refuser de porter plainte, la victime peut influencer les décisions de la justice de deux manières : a) la façon dont elle est perçue par les protagonistes du système, à savoir la police, le procureur, le juge et les jurés; b) sa conduite comme témoin. Prenant cela comme point de départ, les recherches ont tenté 
d'identifier les caractéristiques qui ont, ou qui peuvent avoir une influence prépondérante sur le processus et les décisions de la justice. Deux séries de caractéristiques furent examinées puisqu'elles sont apparues au départ comme intimement liées à l'issue finale de n'importe quel procès : 1) Les attributs de la victime : ceux-ci comportent les traits socio-démographiques tels que l'âge, le sexe, l'origine ethnique, la profession ou l'occupation, la classe sociale, le statut et la respectabilité. Ils incluent également les attributs de la personnalité tels que les perturbations émotionnelles ; les traits physiques tels que l'attrait et le charme; et les circonstances du crime telles que la relation criminel-victime, l'importance de la douleur infligée ou du dommage subi, etc. 2) Les caractéristiques relatives au comportement de la victime : ici on fait généralement la distinction entre la conduite de la victime avant et durant la commission du délit (mauvaise conduite, antécédents criminels, narcomanie ou alcoolisme, vie sexuelle, provocation, précipitation, participation, etc.) et le comportement de la victime lors du procès en qualité de plaignant et de témoin.

Quoique les recherches sur ce sujet ne soient ni suffisantes ni satisfaisantes, les conclusions des études faites à date permettent de croire que la victime joue un rôle significatif (bien qu'informel) dans le processus décisionnel. Parmi les études à noter on doit retenir tout particulièrement celles menées aux États-Unis par Wolfgang et Riedel $(1973,1978)$. Les auteurs ont examiné l'imposition discrétionnelle de la peine capitale dans les cas du viol tout en attirant l'attention sur les disparités raciales. Après avoir analysé vingt-huit variables légales et extra-légales ayant trait au délit, à l'auteur et à la victime, ils ont constaté que la seule variable qui s'est avérée statistiquement significative est celle des hommes noirs violant des femmes blanches.

De la politique préventive axée sur le criminel à la politique axée sur la victime

La politique préventive traditionnelle qui visait à réduire la criminalité par le moyen de la punition ou de la réhabilitation, était axée sur le criminel. Le scepticisme actuel à l'égard de l'efficacité de la peine et de la réhabilitation comme moyens de prévention a mis en évidence la nécessité de trouver des techniques nouvelles. Les stratégies préventives de ce type soulignent le lien étroit entre les différents délits et les opportunités de les com- 
mettre et préconisent, comme moyen le plus efficace de réduire la criminalité, le durcissement des cibles. Contrairement à l'approche traditionnelle qui visait à changer le criminel ou contrôler son comportement, on s'efforce de changer les situations et de réduire les tentations que suscitent les occasions propices à la commission de certains délits. Or, ces tentations, ces opportunités et ces occasions sont souvent créées par les victimes potentielles. Il est donc possible de contrôler le facteur opportunité par des mesures appropriées prises par les victimes potentielles et par la police. Une politique préventive axée sur la victime met en évidence des mesures protectrices, défensives et des précautions que les cibles potentielles pourraient adopter afin de rendre la commission du délit plus difficile et moins rentable. Ceci promet d'être l'un des domaines de la criminologie appliquée auquel la victimologie pourrait faire une contribution importante et valable.

\section{Conclusions}

Durant les dix dernières années, l'intérêt de la victimologie s'est déplacé de la victimisation primaire à la victimisation secondaire, c'est-à-dire aux mauvais traitements que reçoivent les victimes aux mains de la justice. La victimologie a abandonné sa préoccupation presque exclusive du rôle causal de la victime pour devenir soucieuse des droits et des besoins de la victime. On ne parle plus de la responsabilité fonctionnelle de la victime mais de la responsabilité et des obligations sociales envers la victime. Il est done facile de comprendre pourquoi les changements majeurs des dernières années concernant principalement le domaine appliqué et plus particulièrement les champs tels que le dédommagement, les services aux victimes, etc. Les années 70 furent caractérisées par la « redécouverte» de la victime et ont été masquées par une forte réaffirmation de ses droits et de la responsabilité de la société à son égard. Par contre, la présente décennie n'a pas apporté de percées ou de développements spectaculaires sur le plan de la théorie victimologique et les espoirs qu'elle puisse transformer l'étiologie criminelle ; soit une étude statique en une étude dynamique, ne se sont pas encore réalisés.

Mais quoi que furent les développements dans le domaine appliqué, un certain progrès fut accompli sur le plan théorique en ce qui a trait notamment à l'étude de la victimisation; la mesure 
de son ampleur et de son impact, du degré de crainte du crime parmi la population, l'effort de compréhension visant à préciser pourquoi une proportion significative des victimes ne signalent pas l'événement à la police, etc.

Les tentatives d'élaborer des typologies de victimes, qui furent parmi les premières préoccupations des pionniers de la victimologie, ont été remplacées par un intérêt croissant pour l'étude de certains groupes qui, à cause d'une vulnérabilité spécifique, constituent pour les criminels des cibles privilégiées et des proies faciles.

Aussi, durant les dernières années, nos connaissances victimologiques ont été enrichies par des contributions importantes faites par des psychologues dont les études ont abouti à une meilleure compréhension de l'influence qu'exerce la victime, ses caractéristiques et sa conduite, sur les décisions de la justice. Ces études ont démontré le rôle important que joue la victime au moment où le délit est signalé et jusqu'à la décision finale concernant le cas. Elles indiquent que l'influence de la victime est plus forte dans les phases préliminaires du procès (arrestation, poursuite) que dans les étapes ultérieures (prononcé de la sentence ou décision d'accorder la libération conditionnelle).

Un autre domaine où un progrès certain a été fait et auquel ont contribué également les psychologues est celui des perceptions qu'ont les criminels et le public en général, des victimes. La recherche sur ce sujet pourrait éventuellement accroître nos connaissances et notre compréhension des processus de neutralisation, de désensibilisation, et de victimisation. Bien que de plus amples recherches soient nécessaires, nous sommes déjà plus renseignés sur les relations entre l'agresseur et la victime selon les notions de proximité et distance, de rapprochement et d'éloignement et de leur impact sur l'attitude et le comportement du criminel. Nous comprenons davantage l'effet de la stigmatisation sociale et de la légitimation culturelle sur la vulnérabilité à la victimisation de certains individus ou de certains groupes.

Malgré ces progrès théoriques, le chemin est encore long et parsemé d'obstacles. Il semble que plus on en apprend sur les victimes du crime, plus on devient conscient de notre ignorance et du grand besoin de recherches supplémentaires sur ce sujet. 
L'intérêt qu'on porte actuellement à l'étude des caractéristiques et des comportements des victimes est incontestable, mais l'âge d'or de la victimologie est encore très très loin.

\section{BIBLIOGRAPHIE}

AMERICAN BAR ASSOCIATION - SECTION OF CRIMINAL JUSTICE (1977) : Victims of Crime or Victims of Justice? Proceedings of the Annual Meeting held on August 10, 1977 in Chicago, Illinois - Introduction by B. James George, Jr. Washington (D.C.), ABA.

AMIR, M. (1967) : «Victim precipitated forcible rape 》, Journal of Criminal Law, Criminology and Police Science, V. 58, No. 4, p. 493.

AMIR, M. (1971) : Patterns of Forcible Rape, Chicago, Univ. of Chicago Press.

AROMAA, K. (1973) : Victimization to Violence : A Gallup Survey, Paper presented to the First International Symposium on Victimology (2 - 6 September, 1973), Jerusalem, manuscript.

BANDINI, T. and FILAURO, F. (1964) : I delitti contro la persona dell'omosessuale, Medicina legale e delle Assicurazioni, Vol. 12, No. 4, p. 697-717.

BENDER, B. (1976) : Self-chosen Victim : Scapegoat Behavior Sequential to Battering, Child Welfare, Vol. 55, p. 417-422.

BERSHEID, E. and WALSTER, E. (1969) : Interpersonal Attraction, Reading (Mass.), Addison-Wesley.

BIDERMAN, A.D. et al (1967) : Report on a Pilot Study in the District of Columbia on Victimization and Attitudes Toward Law Enforcement, Field Survey I of the President's Commission on Law Enforcement and the Administration of Justice, Washington (D.C.), U.S. Government Printing Office.

BLACK, D.J. (1970) : Production of Crime Rates, American Sociological Review, Vol. 35, p. 733-748.

BLUMENTHAL, M. et al (1972) : Justifying Violence : Attitudes of American Men, University of Michigan, Institute of Social Research.

CALHOUN, L.G., J.W. SELBY and L.J. WARRING (1976) : Social Perception of the Victim's Causal Role in Rape : An Explanatory Examination of Four Factors, Human Relations, Vol. 29, No. 6, p. 517-526.

CIALDINI, R.B. et al (1976) : *Victim Derogation in the Lerner Paradigm. Just World or Just Justification? * Journal of Personality and Social Psychology, V. 33, No. 6, p. 719-724.

CLARK, L. and D. LEWIS, Rape : The Price of Coercive Sexuality, Toronto, Women's Press.

COHEN, Y. (1974) : Help For Victims of Robbery $-T$ wo Researches, Paper presented at the First International Symposium on Victimology, Jerusalem, September 2 - 6, 1973. 
CONKLIN, J. (1971) : Dimensions of Community Response to the Crime Problem, Social Problems, Vol. 18 (Winter), p. 373-384.

CONKLIN, J. (1975) : The Impact of Crime, New York, MacMillan.

COOPER, H.H.A. (1976) : 4 The Terrorist and the Victim 》, Victimology, V. 1, No. 2, p. 229-239.

CORMIER, B. (1966) : On the History of Men and Genocide, Canadian Medical Association Journal, V. 94, p. 276-291.

CRESSEY, D.R. (1953) : Other People's Money, New York, The Free Press of Glencoe. Edited and Reprinted as "The Criminal Violation of Financial Trust , in : Criminal Behavior Systems : A Typology, Edited by Marshall B. Clinard \& Richard Quinney, New York, Holt, Rinehart and Winston, Inc., 1967.

CURTIS, L. (1976) : Toward a Theory of Response to Rape : Some Methodological Considerations, in : E. Viano (ed.) Victims and Society, Washington, Visage Press, p. 220-229.

DADRIAN, V.N. (1972) : The Structural-Functional Component of Genocide : A Victimological Approach to the Armenian Case, Paper presented to the Second Interamerican Congress of the American Society of Criminology, Caracas, Venezuela.

DADRIAN, V.N. (1976) : The Victimization of the American Indian, Victimology, Vol. 1, No. 4, p. 517.537.

DINITZ, S. et al (1976) : The Exploitation Matrix in a Juvenile Institution, International Journal of Criminology and Penology, Vol. 4, p. 259270.

DINITZ, S., S. MILLER and C. BARTOLLAS (1973) : Inmate Exploitation : A Study on the Juvenile Victim, Paper presented to the First International Symposium on Victimology, Jerusalem, September 2 - 6, 1973.

DOBASH, R.E. and R.P. DOBASH (1977) : Wives : The Appropriate Victims of Marital Violence, Victimology, Vol. 2, Nos. 3/4, p. 426-442.

DOERNER, W.G. (1978) : An Examination of the Alleged Latent Effects of Victim Compensation Programs Upon Crime Reporting, LAE Journal of the American Criminal Justice Association, Vol. 41, No. 1 , p. 71.76.

DOERNER, W.G. (1978) : A Quasi-experimental Analysis of Selected Canadian Victim Compensation Programs, Canadian J. of Criminology and Corrections, Vol. 20, No. 3, p. 239-252.

DOERNER, W.G., M.S. KNUDTEN and A. MEADE (1976) : An Analysis of Victim Compensation Programs as a Time-Series Experiment, Victimology, Vol. 1, p. 235-262.

DRAPKIN, I. (1976) : The Prison Inmate as Victim, Victimology, Vol. 1, No. 1 , p. $98-106$.

DYNES, R. and E.L. QUARANTELLI (1974) : Organizations as Victims in Mass Civil Disturbances, in : Drapkin, I. and Viano, E. (eds.) Victimology, Lexington, Lexington Books.

ENNIS, P.H. (1967) : Crime, Victims, and the Police, Transaction, June, Vol. 4 , p. 36-44. 
ENNIS, P.H. (1967) : Criminal Victimization in the United States : A Report of a National Survey, Washington, U.S. Gov't. Printing Office.

FATTAH, E.A. (1967) : « Towards a Criminological Classification of Victims \$, The International Journal of Criminal Police, N. 209.

FATTAH, E.A. (1971) : La victime est-elle coupable? Le rôle de la victime dans le meurtre en vue de vol, Montréal, Les Presses de l'Université de Montréal, $259 \mathrm{p}$.

FATTAH, E.A. (1976) : The Use of the Victim as an Agent of Self Legitimization : Toward a Dynamic Explanation of Criminal Behavior, Victimology, Vol. 1, No. 1, p. 29-53.

FATTAF, E.A. et A. RAIC (1970) : L'alcool en tant que facteur victimogène, Toxicomanies, Vol. 3, No. 2, p. 143-173.

FEENEY, D.G. (1973) : Pity the Criminal Less, More His Innocent Victim, Canadian Society of Forensic Science Journal, Vol. 6, No. 1, March.

FEINBERG, N. (1977) : The Emotional and Behavioral Consequences of Violent Crime on Elderly Victims, Ph.D., University of Pittsburg, $306 \mathrm{p}$.

FELDMAN-SUMMERS, S. and K. LINDNER (1976) : Perceptions of Victims and Defendants in Criminal Assault Cases, Criminal Justice and Behavior, Vol. 3, No. 2, p. 135-150.

FLYNN, E.E. (1976) : The Second International Symposium on Victimology, Victimology, Vol. 1, No. 4, p. 573-579.

FLYNN, E.E. (1977) : Report of the Discussion Sections of the Second International Symposium on Victimology, Victimology, Vol. 2, No. 1, p. $32-48$.

FORSTEN, R.C. (1976) : Criminal Victimization of the Aged : The Houston Model Neighborhood Area, Victimology, Vol. 1, No. 2, p. 316-318.

FORSTEN, R.C. and J. KITCHENS (1975) : Criminal Victimization of the Aged : The Houston Model Neighborhood Area, School of Community Service - North Texas State University, Denton, Texas.

FULERO, S.M. and CH. DELARA (1976) : Rape Victims and Attributed Responsibility : A Defensive Attribution Approach, Victimology, Vol. 1, No. 4, p. 551-563.

FULIER, D. and T. ORSAGH (1977) : Violence and Victimization Within a State Prison System, Criminal Justice Review, Vol. 2, No. 2, p. 35-55.

FURSTENBERG, Jr., F. (1971) : Public Reaction to Crime in the Streets, The American Scholar, 40(4), p. 601-610.

GAROFALO, J. (1977) : Public Opinion About Crime : The Attitudes of Victims and Non-Victims in Selected Cities, Washington (D.C.), Department of Justice.

GELLES, R.J. (1974) : The Violent Home : A Study of Physical Aggressions Between Husbands and Wives, Beverly Hills : Sage Publications.

HAHN, P.H. (1976) : Crimes Against the Elderly - A Study in -Victimology, Santa Cruz, California : Davis Publishing Company. 
HALL, Donald J. (1975) : The Role of the Victim in the Prosecution and Disposition of a Crime Case, Vanderbilt Law Review, Vol. 28, No. 5, p. 931-985.

HOWITT, D. (1977) : Situational and Victim's Characteristics in Simulated Penal Judgments, Psychological Reports, Vol. 40, p. 55-58.

HAWKINS, R.O. (1973) : Who Called the Cops? Decision to Report Criminal Victimization, Law and Society Review, Vol. 7, No. 3, p. 427-444.

INTERNATIONAL ASSOCIATION OF CHIEFS OF POLICE (1965) : Training Manual.

INTERNATIONAL STUDY INSTITUTE ON VICTIMOLOGY (1975) : Conclusions and Recommendations, Victimology, Vol. 1, No. 1, p. 130-152.

JENNINGS, M.A. (1976) : The Victim as Criminal : A Consideration of California's Prostitution Law, California Law Review, Vol. 64, No. 5, p. $1235-1284$.

JOHNSON, P.H. et al (1973) : The Recidivist Victim, Criminal Justice Monograph, Huntsville, Texas : Institute of Contemporary Corrections and the Behavioral Sciences.

JONES, C. and E. ARONSON (1973) : Attribution of Fault to a Rape Victim as a Function of Respectability of the Victim, $J$. of Personality and Social Psychology, Vol. 26, No. 3, p. 415-419.

KEMPE, H.C. (1971) : Pediatric Implications of the Battered Baby Syndrome, Archives of Disease in Childhood, Vol. 46, p. 28-37.

KLEINMAN, F. and D.S. DAVID (1973) : Victimization and Perception of Crime in a Ghetto Community, Criminology, Vol. 11 (Nov.), p. 307-343.

LEJEUNE, R. and N. ALEX (1973) : On Being Mugged : The Event and Its Aftermath, Urban Life and Culture, Vol. 2, No. 3, Oct., p. 259-282.

LERNER, M.J. : Social Psychology of Justice and Interpersonal Attraction, in : T. Huston (ed.) Foundations of Interpersonal Attraction, New York, Academic Press.

LEWIS, P.W. and H.W. MANNLE (1978) : Race and the Death Penalty : the Victim's Influence, J. of the American Criminal Justice Association, Vol. 41, No. 1, p. 47-49.

LIND, B.B. : A Study of Injured Victims of Violence in Oslo, Norway, Manuscript, 11 pages.

LIND, B.B. (1969) : Injured Victims of Violence in Oslo, Nordisk Tidsskrift for Kriminalvidenskab, Vol. 57, Nos. 3/4, p. 207-222.

MARTIN, C.A. and A.S. REBAN (1976) : Criminal Victimization of the Aged in Texas, North Texas State University - Denton, Texas.

MCDONALD, W.F. (1976) : Criminal Justice and the Victim, Vol. VI. Sage Criminal Justice System Annuals, Beverly Hills, Sage Publications.

McINTYRE, J. (1967) : Public Attitudes Toward Crime and Law Enforcement, The Annals, Vol. 374, November, p. 33-46. 
MENDELSOHN, B. (1956) : *Victimologie \$, Revue internationale de criminologie et de police technique, Vol. 10, No. 2, avril-juin.

MENDELSOHN, B. (1968) : Le rapport entre la victimologie et le problème de génocide : pour un code de prévention du génocide, Etudes internationales de psycho-sociologie criminelle, Vol. 14, No. 15, p. 47-53.

MEYERS, M.A. (1977) : The Effects of Victim Characteristics on the Prosecution, Conviction and Sentencing of Criminal Defendants, Ph.D. dissertation, Indiana University, University Microfilms.

MILGRAM, S. (1974) : Obedience to Authority, New York, Harper and Row.

MILLER, M. and J. HEWITT (1978) : Conviction of Defendant as a Function of Juror-Victim Racial Similarity, Journal of Social Psychology, Vol. 105, No. 1, June, p. 159.

MILLER, W. (1973) : Ideology and Criminal Justice Policy : Some Current Issues, J. of Criminal Law and Criminology, Vol. 64, No. 2, p. 141-154.

MILOWEE, I. and R.S. LOURIE (1964) : The Child's Role in the Battered Child Syndrome, J. of Pediatrics, Vol. 65, p. 1079-1081.

NOBLE, J.P. (1975) : Report on a Seminar on $\times$ Women as the Victims of Crime s, Held in Canberra, April 16-19, Australian Institute of Criminology.

OCHBERG, R. (1977) : The Victim of Terrorism - Psychiatric Considerations, Paper presented at an International Seminar on Dimensions of Victimization in the Context of Terroristic Acts, Evian, France.

REDL, F. and D. WEINMAN (1951) : Children Who Hate, New York, The Free Press.

SAGARIN, E. and D.E.J. MacNAMARA (1975) : The Homosexual as a Crime Victim, International J. of Criminology and Penology, Vol. 3, No. 1, p. 13-15.

SCACCO, Jr., A.M. (1975) : Rape in Prison, Springfield, Ill., Charles C. Thomas.

SCHAFER, S. (1968) : The Victim and His Criminal : A Study in Functional Responsibility, New York, Random House.

SCHNEIDER, H.J. (1975) : Victimology : Science of Crime Victims (Viktimologie : Wissenschaft vom Verbrechens-Opfer), Tubingen, J.C.B. Mohr.

SCHRAMM, E. and K. KAISER (1962) : Der homosexuelle mann als Opfer von kapitalverbrechen, Kriminalistik, 16, 6, p. 255-260.

SCHWENDINGER, H. and J. SCHWENDINGER (1967) : Delinquent Stereotypes of Probable Victims, in : M.W. Klein (ed.) Juvenile Gangs in Context, Prentice-Hall, Reprinted in Cressey, D. and Ward, D. (Eds.), Delinquency, Crime and Social Process (1969), New York, Harper and Row, p. 474-487.

SCROGGS, J.R. (1976) : Penalties for Rape as a Function of Victim Provocativeness, damage and resistance, J. of Applied Social Psychology, Vol. 6, No. 4, p. 360-368. 
SICHER, L. (1936) : The Murdered One, Too Is Guilty, International J. of Individual Psychology, Fourth Quarter, Vol. II, No. 4.

SINGLETON, R. and S. HOFACRE (1976) : Effects of Victim's Physical Attractiveness on Juridic Judgments, Psychological Reports, August, Vol. 39, No. 1, p. 73-74.

SKOGAN, W.G. (1976) : Citizen Reporting of Crime : Some National Panel Data, Criminology, Vol. 13, No. 4, p. 535-549.

SMITH, D.L. : The Aftermath of Victimization : Fear and Suspicion, Manuscript, Atlanta (Georgia), Emory University.

SMITH, R.E., J.P. KEATING, R.K. HESTER and H.E. MITCHELL (1976) : Role and Justice Consideration in the Attribution of Responsibility to a Rape Victim, $J$. of Research in Personality, Sept., Vol. 10, No. 3, p. 343-357.

STEINMETZ, S.K. (1977) : The Battered Husband Syndrome, Victimology, Vol. 2, Nos. $3 / 4$, p. 499-509.

STEINMETZ, S.K. and M.A. STRAUS (1973) : The Family as Cradle of Violence, Society, (Sept./Oct.) 54-56.

STRAUS, M.A. (1976) : Sexual Inequality, Cultural Norms, and WifeBeating, Victimology, Vol. 1, No. 1, p. 54-70.

SUTHERLAND, E. (1937) : The Professional Thief, The University of Chicago Press.

SYKES, G. and D. MATZA (1957) : Techniques of Neutralization : A Theory of Delinquency, American Sociological Review, Vol. 22, p. $664-670$.

THRASHER, F.M. (1947) : The Gang, Chicago, University of Chicago Press.

U.S. CONGRESS - SELECT COMMITTEE ON AGING (1977) : In Search of Security : A National Perspective on Elderly Crime Victimization, Washington (D.C.), U.S. Gov't. Printing Office.

VIANO, E. (1976) : Victims and Society, Washington (D.C.), Visage Press.

VON HENTIG, H. (1948) : The Criminal and His Victim, New Haven, Yale University Press.

WALleR, I. (1976) : Victim Research, Public Policy and Criminal Justice, Victimology, Vol. 1, No. 2, p. 240-252.

WEISS, K. and S. BORGES (1973) : Victimology and Rape : The Case of the Legitimate Victim, Issues in Criminology, 8, p. 71-115.

WERFEL, F. (1920) : Nicht der Mörder, der Ermordete ist Schuldig, Munich, Kurt Wolft Verlag.

WILLIAMS, K.M. (1976) A : The Effects of Victim Characteristics on the Disposition of Violent Crimes, in : W. McDonald (ed.), Criminal Justice and the Victim, Beverly Hills, Sage Publications, p. 177-213.

WILLIAMS, K.M. (1976) B : The Victim and Decision-Making by the Criminal Justice System, Victimology, Vol. 1, No. 1, p. 161-162.

WITSCHI, H. (1961) : Das Homosexuellemilieu als Verbrechensquelle, Kriminalistik, 15,4 , p. 145. 
WOLFGANG, M.E. (1957) : *Victim-Precipitated Criminal Homicide *, J. of Criminal Law, Criminology and Police Science, Vol. 48, No. 1, p. 1-11.

WOLFGANG, M.E. (1958) : Patterns in Criminal Homicide, Philadelphia, University of Pennsylvania Press.

WOLFGANG, M.E. (1978) : The Death Penalty : Social Philosophy and Social Science Research, Criminal Law Bulletin, Vol. 14, No. 1, Jan.-Feb., p. 18-33.

WOLFGANG, M.E., A. KELLY and H.C. NOLDE (1962) : Comparisons of the Executed and the Commuted Among Admissions to Death Row, J. of Criminal Law, Criminology and Police Science, Vol. 53, p. 301 .

WOLFGANG, M.E. and M. RIEDEL (1973) : Race, Judicial Discretion, and the Death Penalty, The Annals of the American Academy of Political and Social Science, Vol. 407, p. 119-133.

WOLFGANG, M.E. and R. STROM (1956) : The Relationship Between Alcohol and Criminal Homicide, Quarterly J. of Studies on Alcohol, Vol. 17.

ZAHN, M.A. (1975) : The Female Homicide Victim, Criminology, Vol, 13, No. 3. Nov., p. $400-415$.

ZAHN, M.A. and M. BENCIVENGO (1974) : Violent Death : A Comparison Between Drug Users and Non-Drug Users, Addictive Diseases : An International Journal, Vol. 1, No. 3.

ZAHN, M.A. and M. BENCIVENGO (1975) : Murder in a Drug Using Population, in : Riedl, M., Thornbury, $\mathrm{T}$. (Eds.), Crime and Delinquency : Dimension of Deviance, New York, Praeger.

ZIEGENHAGEN, E.A. (1976) : The Recidivist Victim of Violent Crime, Victimology, Vol. 1, No. 4, p. 538-550. 